\title{
PETS2009 and Winter-PETS 2009 results: a combined evaluation
}

Book or Report Section

Accepted Version

Ellis, A., Shahrokni, A. and Ferryman, J. M. (2010) PETS2009 and Winter-PETS 2009 results: a combined evaluation. In: 2009 Twelfth IEEE International Workshop on Performance Evaluation of Tracking and Surveillance. IEEE. ISBN 9781424455034 doi: https://doi.org/10.1109/PETSWINTER.2009.5399728 Available at https://centaur.reading.ac.uk/17435/

It is advisable to refer to the publisher's version if you intend to cite from the work. See Guidance on citing.

To link to this article DOI: http://dx.doi.org/10.1109/PETS-WINTER.2009.5399728

Publisher: IEEE

All outputs in CentAUR are protected by Intellectual Property Rights law, including copyright law. Copyright and IPR is retained by the creators or other copyright holders. Terms and conditions for use of this material are defined in the End User Agreement.

www.reading.ac.uk/centaur

\section{CentAUR}


Central Archive at the University of Reading

Reading's research outputs online 


\title{
PETS2009 and Winter-PETS 2009 Results: A Combined Evaluation
}

\author{
A.Ellis, A.Shahrokni and J.M.Ferryman \\ Computational Vision Group \\ School of Systems Engineering \\ University of Reading \\ Whiteknights, Reading, RG6 6AY, UK \\ \{a.1.ellis $\mid$ a.shahrokni| j.m.ferryman\}@ reading.ac.uk
}

\section{Datasets and Ground Truth}

\begin{abstract}
This paper presents the results of the crowd image analysis challenge of the Winter PETS 2009 workshop. The evaluation is carried out using a selection of the metrics developed in the Video Analysis and Content Extraction (VACE) program and the CLassification of Events, Activities, and Relationships (CLEAR) consortium [13]. The evaluation highlights the detection and tracking performance of the authors' systems in areas such as precision, accuracy and robustness. The performance is also compared to the PETS 2009 submitted results.
\end{abstract}

\section{Introduction}

This paper discusses the objective evaluation of the submitted results by contributing authors of the two PETS 2009 and Winter PETS 2009 workshops on the challenges defined on the PETS2009 crowd dataset [12] .

The theme of the Winter PETS 2009 is multi-sensor event recognition in crowded public areas. As part of this workshop a challenge was set to evaluate an approach to one or more of people counting and density estimation, tracking, and flow estimation and event recognition, and to report results based on annotated datasets made available on the workshop website [1]. In this paper the focus is tracking and people counting challenges due to the fact that the majority of the submitted evaluations and papers were dedicated to these tasks.

In the remainder of this paper, the dataset and the ground truth annotation details are presented in Section 2. A brief description of the evaluation methodology follows in Section 3, and analytic discussion of the overall performances is provided in Section 4. Concluding remarks are given in Section 5.

\subsection{Datasets}

Three datasets were recorded for the workshop at Whiteknights Campus, University of Reading, UK. Further details of these datasets may be found in Ferryman and Shahrokni [12]. The datasets comprise multi-sensor sequences containing crowd scenarios with increasing scene complexity. Dataset S1 concerns person count and density estimation. Dataset S2 addresses people tracking. Dataset S3 involves flow analysis and event recognition. In this paper the first two datasets are the focus.

\subsection{Ground Truth}

The ground truth was obtained for a subsampled set of frames for each sequence with the average sampling frequency being 1 frame in every 3 frames.

The ground truth for people counting was generated by manually counting the people in the specified regions and those that cross the entry and exit lines at each sampled frame.

The ground truth annotation simultaneously defines bounding boxes in all views corresponding to a person, by locating its $3 \mathrm{D}$ position on a discrete grid. The grid is defined as cells of 72 in width and 132 in height on the ground plane, which corresponds to an area of $24 \times 44$ metres. Errors in calibration due to the approximation of the ground surface as a plane, in addition to radial distortion, and the spatial resolution of the annotation grid defined on the ground plane, are an intrinsic part of this annotation. In the context of the current evaluations, further measures have been considered to take this into account in the evaluation process as described in Section 3. 


\section{Evaluation Methodology}

The evaluation was based on the framework by Kasturi et al. [13], which is a well established protocol for performance evaluation of object detection and tracking in video sequences. These metrics are formally used the Video Analysis and Content Extraction (VACE) program and the CLassification of Events, Activities, and Relationships (CLEAR) consortium. As part of the PETS 2009 workshop, authors of the representative algorithms, submitted their results in XML format using the PETS 2009 published XML Schema available at [1]. These results were evaluated using the following metrics:

\section{Notation}

- $G_{i}^{t}$ denotes $i^{t h}$ ground-truth object in frame $t ; G_{i}$ denotes the $i^{t h}$ ground-truth object at the sequence level; $N_{\text {frames }}$ is the number of frames in the sequence

- $D_{i}^{t}$ denotes the $i^{t h}$ detected object in frame $t ; D_{i}$ denotes the $i$ th detected object at the sequence level

- $N_{G}^{t}$ and $N_{D}^{t}$ denote the number of ground-truth objects and the number of detected objects in frame $t$, respectively; $N_{G}$ and $N_{D}$ denote the number of unique ground-truth objects and the number of unique detected objects in the given sequence, respectively

- $N_{\text {frames }}^{i}$ refers to the number of frames where either ground-truth object $\left(G_{i}\right)$ or the detected object $\left(D_{i}\right)$ existed in the sequence

- $N_{\text {mapped }}$ refers to sequence level detected object and ground truth pairs, $N_{\text {mapped }}^{t}$ refers to frame $t$ mapped ground truth and detected object pairs

- $m_{t}$ represents the missed detection count, $\left(f p_{t}\right)$ is the false positive count, $c_{m}$ and $c_{f}$ represent respectively the cost functions for missed detects and false positives, and $c_{s}=\log _{10} I D-S W I T C H E S_{t}$

\subsection{Sequence Frame Detection Accuracy (SFDA)}

SFDA uses the number of objects detected, the number of missed detections, the number of falsely identified objects, and the calculation of the spatial alignment between the algorithm's output for detected objects and that of the ground truthed objects. It is derived from a Frame Detection Accuracy (FDA) measure. The FDA is calculated using a ratio of the spatial intersection and union of an output object and mapped ground truthed objects

$$
\begin{gathered}
\text { OverLapRatio }=\sum_{i=1}^{N_{\text {mapped }}^{t}} \frac{\left|G_{i}^{t} \cap D_{i}^{t}\right|}{\left|G_{i}^{t} \cup D_{i}^{t}\right|} \\
F D A(t)=\frac{\text { OverlapRatio }}{\left[\frac{N_{G}^{t}+N_{D}^{t}}{2}\right]} \\
\text { SFDA }=\frac{\sum_{t=1}^{N_{\text {frames }}} F D A(t)}{\sum_{t=1}^{N_{\text {frames }}} \exists\left(N_{G}^{t} \vee N_{D}^{t}\right)}
\end{gathered}
$$

For this study although the annotation of the ground truth was challenging, as described in Section 2, an overlap threshold of 100 percent for the intersection over union scores, was used.

\subsection{Average Tracking Accuracy (ATA)}

ATA is obtained from the Sequence Track Detection Accuracy (STDA). The STDA is a measure of the tracking performance over all of the objects in the sequence and from this ATA is defined as the sequence track detection accuracy per object. The mapping between ground truth objects and detected objects is performed so as to maximise the measure score. This metric is implemented with a hash function due to the fact that the track correspondence matrix to be mapped is reasonably sparse.

$$
\begin{gathered}
S T D A=\sum_{i=1}^{N_{\text {mapped }}} \frac{\sum_{i=1}^{N_{\text {frames }}}\left[\frac{\left|G_{i}^{t} \cap D_{i}^{t}\right|}{\left|G_{i}^{t} \cup D_{i}^{t}\right|}\right]}{N_{\left(G_{i} \cup D_{i} \neq 0\right)}} \\
A T A=\frac{S T D A}{\left[\frac{N_{G}+N_{D}}{2}\right]}
\end{gathered}
$$

For both detection and tracking metrics in the following descriptions the accuracy metrics provide a measure of the correctness of the detections or tracks. The precision metrics provide the measure of, in the instance where there has been a correct detection or track, how close to the ground truth that detection or track may be.

\subsection{Multiple Object Detection Accuracy (MODA)}

MODA is an accuracy measure that uses the number of missed detections and the number of falsely identified objects. Cost functions to allow weighting to either of these errors are included, however for the sake of both PETS 2009 evaluations they were equally set to 1 .

$$
M O D A=1-\frac{c_{m}\left(m_{t}\right)+c_{f}\left(f_{p_{t}}\right)}{N_{G}^{t}}
$$




\subsection{Multiple Object Detection Precision (MODP)}

MODP gives the precision of the detection in a given frame. Again, with this metric, an overlap ratio is calculated as previously defined in (1), and, in addition to a count of the number of mapped objects, the MODP is defined as:

$$
\operatorname{MODP}(t)=\frac{\text { OverLapRatio }}{N_{\text {mapped }}^{t}}
$$

\subsection{Multiple Object Tracking Accuracy (MOTA)}

MOTA uses the number of missed detections, the falsely identified objects, and the switches in an algorithm's output track for a given ground truth track. These switches are calculated from the number of identity mismatches in a frame, from the mapped objects in its preceding frame.

$$
\operatorname{MOT} A=1-\frac{\sum_{t=1}^{N_{\text {frames }}}\left(c_{m}\left(m_{t}\right)+c_{f}\left(f_{p_{t}}\right)+c_{s}\right)}{\sum_{t=1}^{N_{\text {frames }}} N_{G}^{t}}
$$

\subsection{Multiple Object Tracking Precision (MOTP)}

MOTP is calculated from the spatio-temporal overlap between the ground truthed tracks and the algorithm's output tracks.

$$
\operatorname{MOTP}=\frac{\sum_{i=1}^{N_{\text {mapped }}} \sum_{t=1}^{N_{\text {frames }}^{t}\left[\frac{\left|G_{i}^{t} \cap D_{i}^{t}\right|}{\left|G_{i}^{t} \cup D_{i}^{t}\right|}\right]}}{\sum_{t=1}^{N_{f} \text { rames }} N_{\text {mapped }}^{t}}
$$

In addition to the evaluation of tracking, a simple comparison of the people count per region, against a ground truth count per region for the sampled frames, produced the average percentage error in counting per region, for each sequence.

\section{Evaluation Results}

An analysis of the overall performance, of the submitted results from the benchmark datasets, using the described metrics, is described in this section. The submitted results are diverse in terms of the sequences and views used and therefore it is not possible to draw general comparisons and conclusions about their performance. Nevertheless, the evaluations presented in this section can lead to a helpful insight about the effectiveness of different methodologies. Both people counting and tracking challenges are considered.

\subsection{People Counting}

Figure 1 provides the evaluation of the counting people per region task. Note that the y axis on this graph represents the average error in number of people per frame, where the lower the value, the better the performance per frame. Table 1 gives the corresponding publication reference, for each label, for Figure 1.

Table 1: Labels and publication references for Figure 1

\begin{tabular}{|c|c|}
\hline Label & Reference \\
\hline Chan & {$[10]$} \\
Sharma & {$[15]$} \\
Albiol & {$[3]$} \\
Choudri & {$[11]$} \\
Alahi & {$[2]$} \\
\hline
\end{tabular}

A wide variety of methods have been proposed and tested in this category and from Figure 1 it can been seen that the majority of the methods and their variants have consistent and comparable performance. While the algorithm proposed by Alboil et al. [3] remains a top performer, several methods such as Alahi et al. [2], Chan et al. [10] and Choudri et al. [11] also perform well on the more challenging sequence 14-17. Further details of the variant of each method can be found in their companion workshop paper.

\subsection{Tracking}

The most tested dataset of the two PETS workshops in 2009 remains S2.L1, at time sequence 12.34, for the first camera view. Figure 3 shows how the individual algorithms perform according to various VACE and CLEAR metrics on a single representative camera view. Table 2 gives the corresponding publication reference, for each label, for Figures 3,4 and 5 .

Note that in the case of these metrics, higher values indicate better performance. It is clear that for this sequence, using MODA and MOTA as a measure, Yang et al.'s [16], Breitenstein et al.'s [9] and the linear programming-based method proposed by Berclaz et al. [6] perform strongly at multiple object detection and tracking. For precision in this task, using MODP and MOTP, the systems described by Berclaz et al. [7], as well the dynamic programmingbased method proposed in [6] perform the strongest. Measuring object detection accuracy by frame and sequence using SODA and SFDA metrics, the systems by Berclaz et al. $[7,6]$ outperform others.

Figure 4 shows the median of each metric value, for all the computed views, excluding View 2 which was not provided in the dataset, from each author. Again, the performance measures highlight the linear programming-based 


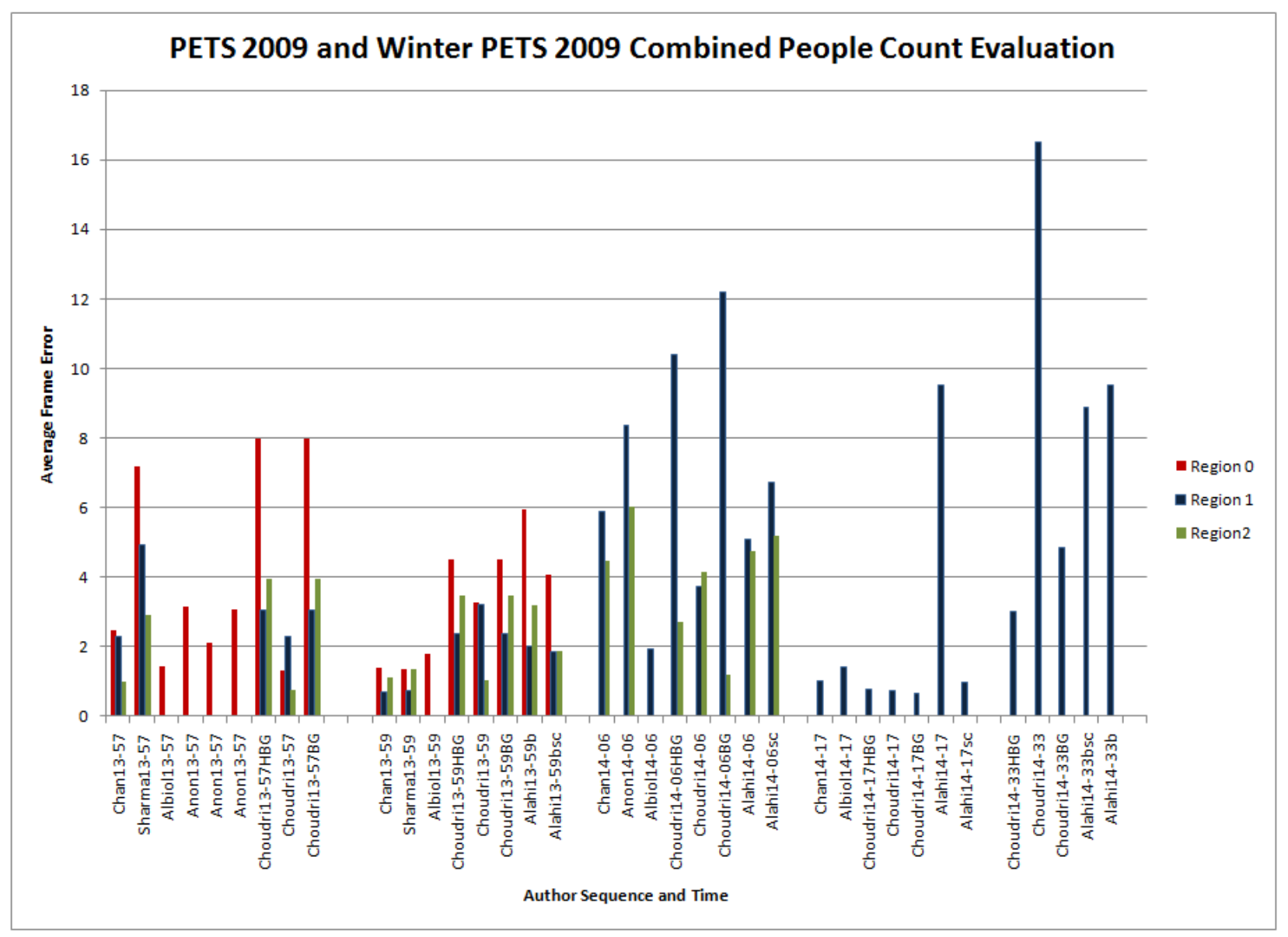

Figure 1: Counting People in Regions.

tracking algorithm [6] for multiple object detection and accuracy. From this figure it can be seen that although there are variations per metric per author, the results for MOTP, MODP, SFDA, and SODA indicate a general consensus of accuracy.

To estimate the consistency of the metrics themselves another evaluation is illustrated here. Figure 2 shows, for each view, the median value of each metric for all authors. It highlights the relationship between five (SODA, SFDA, MODP, MODA, MOTP) of the six metrics used. In addition it indicates the difficulty in detection and tracking from camera View 7. Camera View 2 was also particularly challenging as it was not provided to the authors and the results have be obtained by re-projection from other views or the $3 \mathrm{D}$ information. Overall this Figure shows that the metrics are consistent in their evaluation of performance.

As the final evaluation, a view for each metric which corresponds to the median value of the metric for all authors. The results are shown in Figure 5.

From this Figure a fair overall performance comparison of each algorithm and it variant forms can be inferred. Due to its robustness to outliers, this visualisation gives a clear indication of how different algorithms perform relative to each other. It must be noted that in some cases the accuracy measure can be negative if the number of false positives are high as been seen for MOTA8 in Figure 5.

\section{Conclusion}

It is essential that authors are able to objectively evaluate their detection and tracking algorithms with standardised metrics. The ability to compare results, with others, whether anonymous or not, provides a realistic and encouraging research technique towards advanced, robust, realtime visual systems. In order for authors to achieve this, during the many iterative cycles of algorithm development, an online tool that they may use at their convenience seems sensible. However, in order for such a tool to be completely automated, the complexity of any appropriate XML files 




Figure 2: Median metric values among all authors per view

must be an absolute minimum.

In addition, the use of these metrics and this study provides a mechanism to highlight the strengths of the individual systems, such as accuracy, precision and robustness. It may be used for future decisions for systems placement. For example, those that require a high degree of precision may benefit from techniques described by authors whose systems performed well using precision metrics.

\section{References}

[1] PETS: of Tracking and http://www.cvg.rdg.ac.uk/slides/pets.html.

[2] A. Alahi, L. Jacques, Y. Boursier, and P. Vandergheynst. Sparity-driven people localization algorithm: Evaluation in crowded scenes environments. In Twelfth IEEE International Workshop on Performance Evaluation of Tracking and Surveillance, 2009.
[3] A. Albiol, M. J. Silla, A. Albiol, and J. M. Mossi. Video analysis using corners motion analysis. In Eleventh IEEE International Workshop on Performance Evaluation of Tracking and Surveillance, pages 31-37, 2009.

[4] D. Arsic, A. Lyutskanov, G. Rigoll, and B. Kwolek. Multi camera person tracking applying a graph-cuts based foreground segmentation in a homography framework. In Twelfth IEEE International Workshop on Performance Evaluation of Tracking and Surveillance, 2009.

[5] L. Bazzani, D. Bloisi, and V. Murino. A comparison of multi hypothesis kalman filter and particle filter for multi-target tracking. In Eleventh IEEE International Workshop on Performance Evaluation of Tracking and Surveillance, 2009.

[6] J. Berclaz, F. Fleuret, and P. Fua. Multiple object tracking using flow linear programming. In Twelfth 


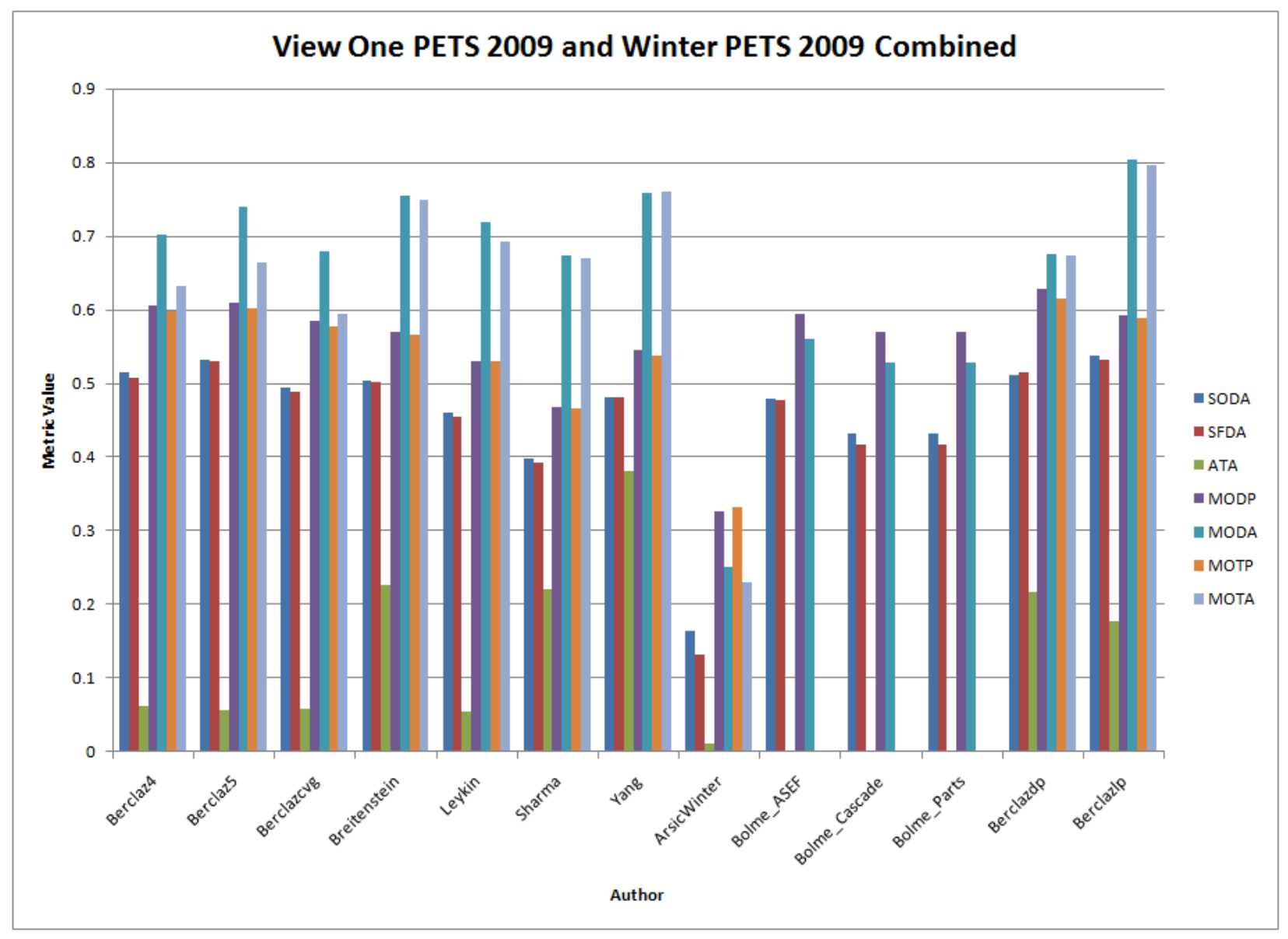

Figure 3: Performance of Authors' Systems Per Metric,Camera View 1, Dataset:S2.L1, Time Sequence: 12.34.

IEEE International Workshop on Performance Evaluation of Tracking and Surveillance, 2009.

[7] J. Berclaz, A. Shahrokni, F. Fleuret, J. M. Ferryman, and P. Fua. Evaluation of probabilistic occupancy map people detection for surveillance systems. In Eleventh IEEE International Workshop on Performance Evaluation of Tracking and Surveillance, pages 55-62, 2009.

[8] D. Bolme, Y. Lui, B. Draper, and J. Beveridge. Simple real-time human detection using a single correlation filter. In Twelfth IEEE International Workshop on Performance Evaluation of Tracking and Surveillance, 2009.

[9] M. D. Breitenstein, F. Reichlin, B. Leibe, E. KollerMeier, and L. van Gool. Markovian tracking-bydetection from a single, uncalibrated camera. In Eleventh IEEE International Workshop on Performance Evaluation of Tracking and Surveillance, pages 71-78, 2009.
[10] A. B. Chan, M. Morrow, and N. Vasconcelos. Analysis of crowded scenes using holistic properties. In Eleventh IEEE International Workshop on Performance Evaluation of Tracking and Surveillance, pages 101-108, 2009.

[11] S. Choudri, J. Ferryman, and A. Badii. Robust background model for pixel based people counting. In Twelfth IEEE International Workshop on Performance Evaluation of Tracking and Surveillance, 2009.

[12] J.Ferryman and A. Shahrokni. An overview of the pets2009 dataset. In Twelfth IEEE International Workshop on Performance Evaluation of Tracking and Surveillance, 2009.

[13] R. Kasturi, D. Goldgof, P. Soundararajan, V. Manohar, J. Garofolo, R. Bowers, M. Boonstra, V. Korzhova, and Jing Zhang. Framework for performance evaluation of face, text, and vehicle detection and tracking in video: Data, metrics, and protocol. Pattern Analy- 


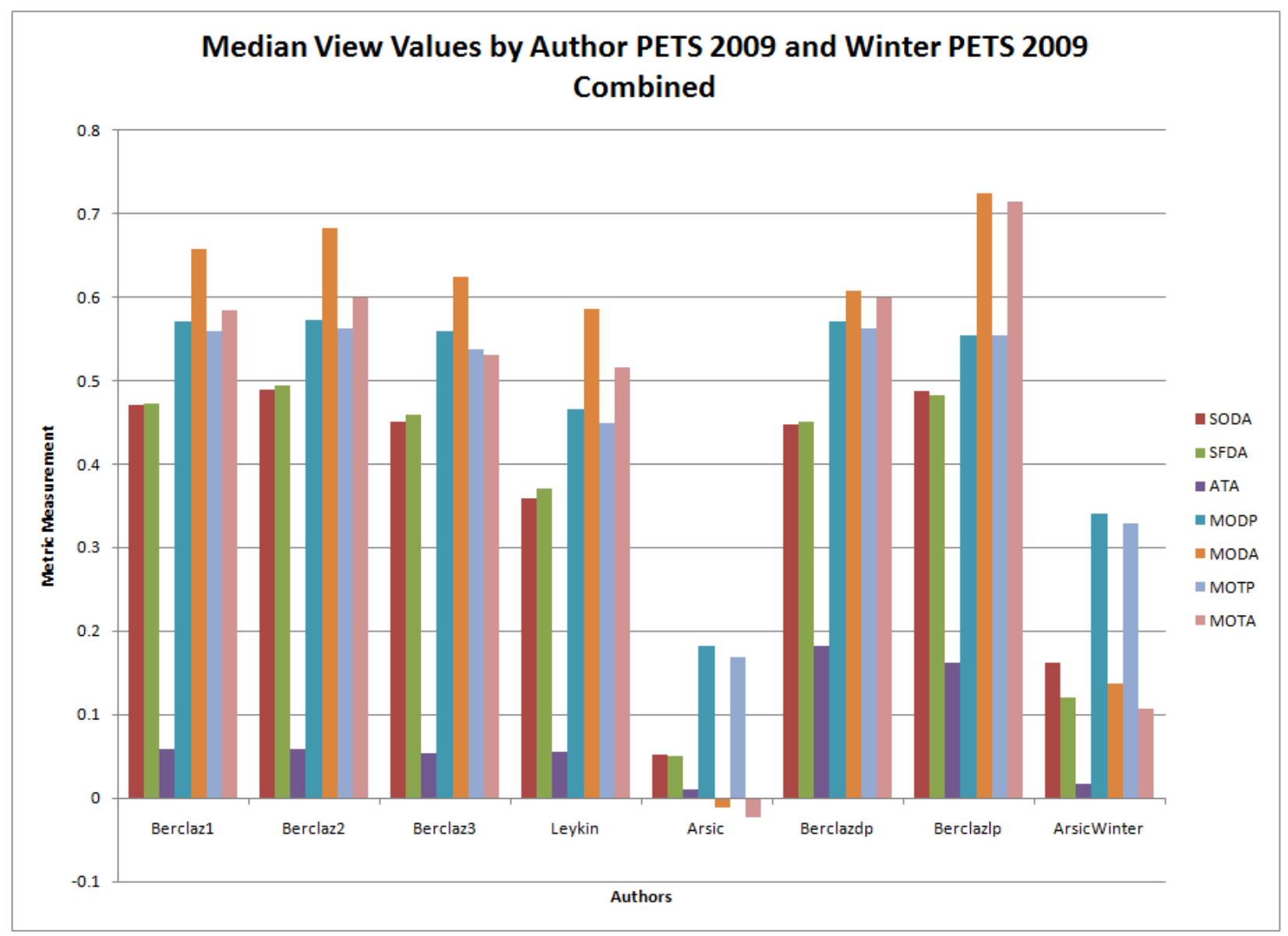

Figure 4: Median metric measurement across all views for different authors, Dataset:S2.L1, Time Sequence: 12.34.

sis and Machine Intelligence, IEEE Transactions on, 31(2):319-336, Feb. 2009.

[14] N. Lehment, D. Arsic, A. Lyutskanov, B. Schuller, and G. Rigoll. Statistical filters for crowd image analysis. In Twelfth IEEE International Workshop on Performance Evaluation of Tracking and Surveillance, 2009.

[15] P. K. Sharma, C. Huang, and R. Nevatia. Evaluation of people tracking, counting and density estimation in crowded environments. In Eleventh IEEE International Workshop on Performance Evaluation of Tracking and Surveillance, pages 39-46, 2009.

[16] J. Yang, Z. Shi, P. Vela, and J. Teizer. Probabilistic multiple people tracking through complex situations. In Eleventh IEEE International Workshop on Performance Evaluation of Tracking and Surveillance, pages 79-86, 2009.
Table 2: Labels and publication references for Figures 3, 4 and 5

\begin{tabular}{|c|c|}
\hline Label & Reference \\
\hline Bazzani & {$[5]$} \\
Berclaz1 & {$[7]$} \\
Berclaz2 & {$[7]$} \\
Berclaz3 & {$[7]$} \\
Breitenstein & {$[9]$} \\
Leykin & not published \\
Sharma & {$[15]$} \\
Yang & {$[16]$} \\
Arsic & {$[14]$} \\
ArsicWinter & {$[4]$} \\
Bolme_ASEF & {$[8]$} \\
Bolme_Cascade & {$[8]$} \\
Bolme_Parts & {$[8]$} \\
Berclazdp & {$[6]$} \\
Berclazlp & {$[6]$} \\
\hline
\end{tabular}




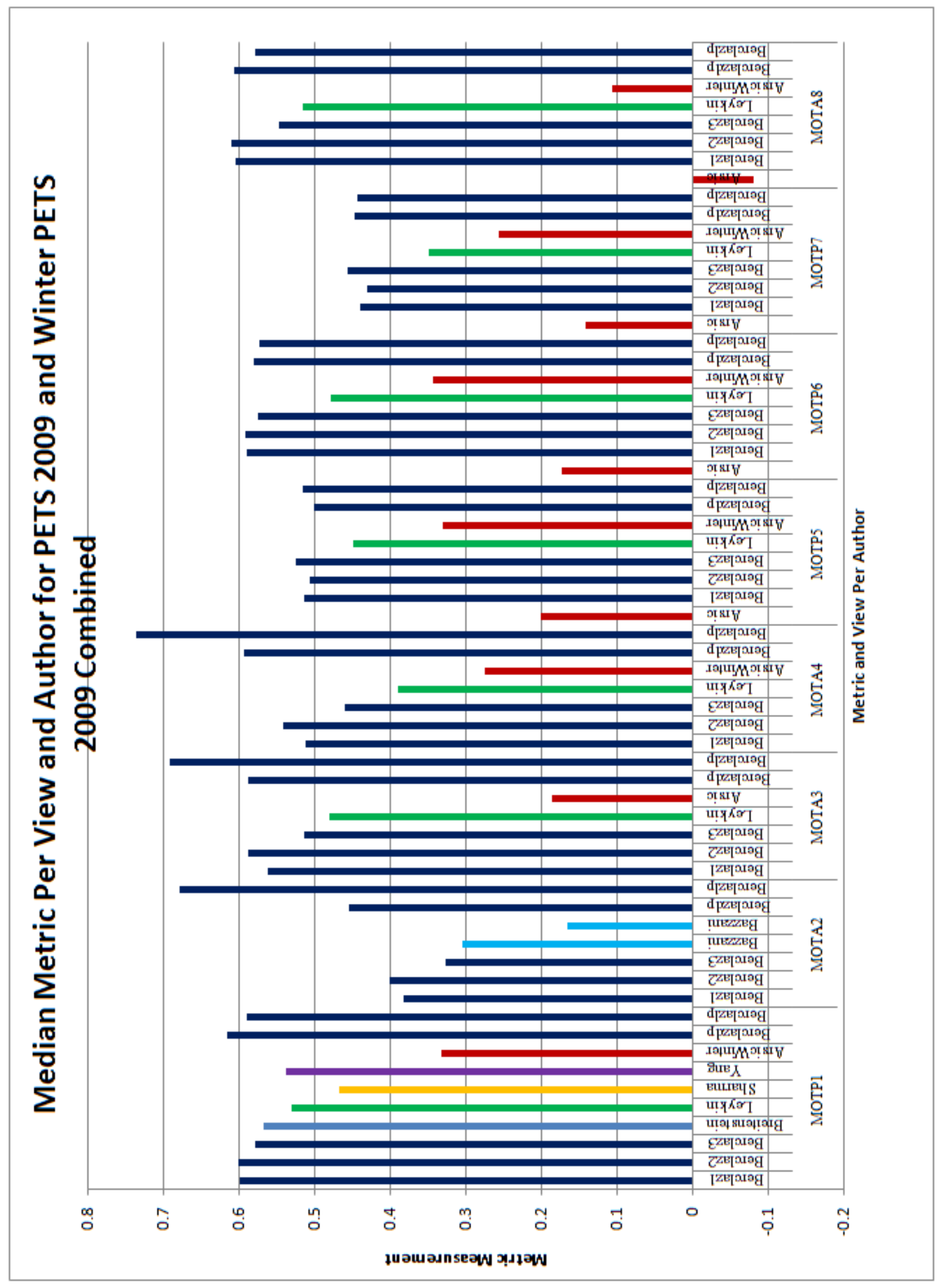

Figure 5: Median metric per view per author. 